\title{
Constructing Human Proteoform Families Using Intact-Mass and Top-Down Proteomics with a Multi-Protease Global PTM Discovery Database
}

\author{
Yunxiang Dai, ${ }^{1,2, \#}$ Katherine E. Buxton, ${ }^{1, \#}$ Leah V. Schaffer, ${ }^{1}$ Rachel M. Miller, ${ }^{1}$ Robert J. Millikin, ${ }^{1}$ \\ Mark Scalf, ${ }^{1}$ Brian L. Frey, ${ }^{1}$ Michael R. Shortreed, ${ }^{1}$ Lloyd M. Smith ${ }^{*}, 1$ \\ ${ }^{1}$ Department of Chemistry, University of Wisconsin, Madison, Wisconsin, United States \\ ${ }^{2}$ Biophysics Graduate Program, University of Wisconsin, Madison, Wisconsin, United States \\ \# These authors contributed equally to this work. \\ *Corresponding author: E-mail: smith@chem.wisc.edu. Phone: (608) 263-2594. Fax: (608) 265-6780.
}

Table of Contents:

Supplementary Experimental Methods

(Materials, Cell Lysis for NeuCode Intact-Mass Proteomics, Gelfree Fractionation, LC/MS Methods for

NeuCode Intact-Mass Proteomics, Bottom-Up Proteomics, Gelfree Fractionation and LC/MS Methods for Top-

Down Proteomics, Intact-Mass Data Deconvolution, Proteoform Family Visualization with Description of

Alterations)

Raw MS Data Files and G-PTM-D Databases

Supplementary Results

(NeuCode Intact-Mass-Only Analysis Using Unpruned Multi-Protease G-PTM-D Database, Integrated Intact-

Mass/Top-Down Analysis Using Uncalibrated Data, Top-Down-Only Analysis Utilizing Top-Down MS1

Spectra as "Label-Free Intact-Mass" Data, Protein Entries in the Patch Database, Histone Proteoforms and

PTMs)

Supplementary Figures

Figure S-1: $\quad$ Lysine Count and Intensity Ratio Distributions of the NeuCode pairs

Figure S-2: $\quad$ Mass Difference Histogram from Experimental-Theoretical Proteoform Comparisons

Figure S-3: $\quad$ Mass Difference Histogram from Experimental-Experimental Proteoform Comparisons

Figure S-4 Molecular Weight Distribution of the 1,207 Identified Proteoforms

Figure S-5: $\quad$ PTM Types Found in the 1,207 Identified Proteoforms

Figure S-6: Histone H3.1t Proteoform Family

Supplementary Tables in Excel File:

Table S-1: $\quad$ PTMs and Artifacts Searched in Building the G-PTM-D Databases

Table S-2: $\quad$ Proteoform Suite Data Analysis Time

Table S-3: $\quad$ List of 5,615 Aggregated Intact-Mass Experimental Proteoforms

Table S-4: New Modifications in the Multi-Protease G-PTM-D Database (Not in UniProt)

Table S-5: $\quad$ Selected ET and EE Mass Differences in Intact-Mass Analyses with Three Different Databases

Table S-6: $\quad$ List of 442 Unique Proteoform Identifications from Intact-Mass-Only Analysis

Table S-7: $\quad$ Summary of Results and Settings for Intact-Mass-Only Analyses Using Three Databases

Table S-8: $\quad$ List of 6,123 Aggregated Experimental Proteoforms (5,309 Intact-Mass) from Integrated Analysis

Table S-9: $\quad$ List of 6,123 Aggregated Experimental Proteoforms (814 Top-Down) from Integrated Analysis

Table S-10: $\quad$ Proteoform Families and Orphans from Integrated Intact-Mass/Top-Down Analysis

Table S-11: $\quad$ Selected ET and EE Mass Differences in Integrated Intact-Mass/Top-Down Analysis

Table S-12: $\quad$ Summary of Results and Settings for Integrated Intact-Mass/Top-Down Analysis

Table S-13: $\quad$ List of 1,207 Unique Proteoform Identifications from Integrated Intact-Mass/Top-Down Analysis

Table S-14: $\quad$ List of Genes Represented by Proteoform Identifications

Table S-15: $\quad$ Gene Ontology Term Analysis of Genes Represented by Proteoform Identifications 


\section{SUPPLEMENTARY EXPERIMENTAL METHODS}

\section{Materials}

- Jurkat cells (TIB-152) were purchased from the American Type Culture Collection (ATCC) (Manassas, VA).

- SILAC RPMI-1640 medium (A2494401), fetal bovine serum (26400036), antibiotic-antimycotic solution (15240062), HEPES buffer (15630080), sodium pyruvate solution (11360070), GlutaMAX (35050061), 100X HALT protease/phosphatase inhibitor cocktail (78441), and methanol (A452) were purchased from Thermo Fisher Scientific (Waltham, MA).

- L-arginine (A5006), DL-dithiothreitol (DTT) (D5545), sodium butyrate (B5887), iodoacetamide (I1149), acetone (270725), unlabeled L-lysine (62840), 10\% sodium dodecyl sulfate (SDS) (71736), and chloroform (319988) were purchased from Sigma-Aldrich Corp. (St. Louis, MO).

- L-lysine:2HCl, ${ }^{13} \mathrm{C}_{6}{ }^{15} \mathrm{~N}_{2}$ (CNLM-291-H) and L-lysine:2HCl, 3,3,4,4,5,5,6,6 $\mathrm{D}_{8}$ (DLM-2641) were purchased from Cambridge Isotope Laboratories, Inc. (Tewksbury, MA).

- $4 \mathrm{M}$ Tris-HCl pH=7.5 (T5575), 10X phosphate buffered saline (PBS) (P0195), and 500 mM EDTA $\mathrm{pH}=8.0$ (E0306) were purchased from Teknova (Hollister, CA).

- Gelfree 8100 12\% Tris-acetate cartridge (42402), Tris-acetate 5X sample buffer (42302), and HEPES running buffer (42202) were purchased from Expedeon (San Diego, CA).

- Acetonitrile (ACN) (AH015-4) was purchased from Honeywell (Morris Plains, NJ).

- Formic acid (11670) was purchased from EMD Millipore (Burlington, MA).

\section{Cell Lysis for NeuCode Intact-Mass Proteomics}

For each replicate NeuCode intact-mass experiment, approximately $10^{7}$ "light" and $10^{7}$ "heavy" labeled Jurkat cells were thawed and lysed separately in $1 \mathrm{~mL}$ buffer containing 4\% (w/v) SDS, $100 \mathrm{mM}$ Tris$\mathrm{HCl} \mathrm{pH}=7.5,10 \mathrm{mM}$ DTT, $10 \mathrm{mM}$ sodium butyrate, $20 \mathrm{mM}$ EDTA, and 1X HALT protease/phosphatase inhibitors. The cells were incubated at room temperature for $10 \mathrm{~min}$ with frequent vortexing, then sonicated in a water bath sonicator (FS20, Fisher Scientific) for 5 min with $20 \mathrm{~s} \mathrm{on/off} \mathrm{intervals.} \mathrm{The}$ lysate was incubated for an additional $30 \mathrm{~min}$ at room temperature, then proteins were alkylated with 20 $\mathrm{mM}$ iodoacetamide for $30 \mathrm{~min}$. Residual iodoacetamide was quenched via a $15 \mathrm{~min}$ incubation with a final concentration of $20 \mathrm{mM}$ DTT. Proteins were precipitated with acetone at $-20^{\circ} \mathrm{C}$ and resuspended in $200 \mu \mathrm{L}$ of $1 \%$ SDS. Proteins from the two NeuCode-labeled samples were then mixed in a 2:1 "light":"heavy" ratio by volume.

\section{Gelfree Fractionation}

Two $\sim 65 \mu \mathrm{L}$ aliquots of this mixed protein sample were added to new $1.7 \mathrm{~mL}$ tubes. Sample buffer $(30$ $\mu \mathrm{L}), 1 \mathrm{M}$ DTT $(8 \mu \mathrm{L})$, and water were added to bring the total volume in each tube up to $150 \mu \mathrm{L}$. The tubes were incubated at $50{ }^{\circ} \mathrm{C}$ for $10 \mathrm{~min}$, then cooled to room temperature. The contents of each tube were fractionated in separate $12 \%$ Tris-acetate Gelfree cartridge channels using the manufacturer's recommended procedure. To prepare the channels, storage buffer was removed and replaced with running buffer. Each of the $150 \mu \mathrm{L}$ samples was loaded, and a standard running method was used to separate the samples into fractions based on molecular weight. Between each step in the method, fractions in the collection chambers of the two channels were combined into a new $2 \mathrm{~mL}$ low-retention tube. The collection chambers were rinsed and replenished with new running buffer. These steps were repeated 11 times for each fraction collection. Throughout the run, the running buffer was changed twice according to the standard procedure. The collected fractions were stored at $-20^{\circ} \mathrm{C}$ or $4^{\circ} \mathrm{C}$ for subsequent sample preparation. Prior to mass spectrometric analysis, SDS was removed from each of the fractions via methanol-chloroform precipitation ${ }^{1}$ and proteins were reconstituted with $16 \mu \mathrm{L}$ of $5 \% \mathrm{ACN}$ and $0.2 \%$ formic acid in water. Intact protein solutions were gently vortexed and centrifuged on a bench-top 
centrifuge for $1 \mathrm{~min}$. Solutions were carefully transferred into HPLC sample vials, leaving behind undissolved substances. Three biological replicates of this experiment were performed.

\section{LC/MS Methods for NeuCode Intact-Mass Proteomics}

All fractions were analyzed by HPLC-ESI-MS (nanoAcquity, Waters and QE-HF Orbitrap, Thermo Fisher Scientific). HPLC separation employed a $100 \times 365 \mu \mathrm{m}$ fused silica capillary microcolumn packed with $20 \mathrm{~cm}$ of $5 \mu \mathrm{m}$ diameter, $1000 \AA$ pore size PLRP-S resin (Agilent) with an emitter tip pulled to approximately $1 \mu \mathrm{m}$ using a laser puller (Sutter Instruments). Proteins were loaded on-column at a flow rate of $500 \mathrm{~nL} / \mathrm{min}$ for $30 \mathrm{~min}$, then eluted at $500 \mathrm{~nL} / \mathrm{min}$ over $67 \mathrm{~min}$ with a gradient of $5 \%$ to $85 \% \mathrm{ACN}$ in $0.2 \%$ formic acid. Full-mass profile scans were performed between 500 and 1,600 $\mathrm{m} / \mathrm{z}$ at a resolution of 240,000. Seven microscans were averaged, using an AGC target of $3 \times 10^{6}$ with a maximum injection time of $200 \mathrm{~ms}$. Source-induced dissociation was set to $15.0 \mathrm{eV}$. Two technical replicate injections of each fraction were performed, yielding a total of 66 raw data files ( 3 biological replicates $\times 11$ fractions $\times 2$ injections).

\section{Bottom-Up Proteomics}

Bottom-up proteomics data were collected previously for five aliquots of Jurkat cell lysate, each of which was digested with a different protease (chymotrypsin, GluC, ArgC, AspN, and LysC). ${ }^{2}$ Methods for cell culture, lysis, FASP (including different digestion conditions for different proteases), peptide fractionation via high-pH reversed-phase liquid chromatography, and LC/MS are described in detail elsewhere. ${ }^{2-3}$ As part of a separate work, this process was repeated to collect MS data for tryptic peptides from unlabeled Jurkat cell lysate. ${ }^{4}$ The only alterations to the aforementioned procedure were as follows: proteins were digested in $50 \mathrm{mM}$ ammonium bicarbonate buffer $(\mathrm{pH}=7.8)$ using a 1:50 trypsin:protein ratio, 10 fractions of peptides were collected (instead of 11 ), and peptides were reconstituted with $5 \% \mathrm{ACN} / 1 \%$ formic acid in water prior to LC/MS.

\section{Gelfree Fractionation and LC/MS Methods for Top-Down Proteomics}

Proteins from label-free Jurkat cell lysate were fractionated by Gelfree as described for the NeuCodelabeled samples, except $\sim 110 \mu \mathrm{L}$ of resuspended protein were fractionated in a single Gelfree channel. Top-down analysis of each of the 11 Gelfree fractions was performed via HPLC-ESI-MS/MS (nanoAcquity, Waters and QE-HF Orbitrap, Thermo Fisher Scientific). The LC method was the same as the intact-mass experiments. MS1 scans were performed between 500 and 1,600 $\mathrm{m} / \mathrm{z}$ at a resolution of 240,000. Seven microscans were averaged, using an AGC target of $1 \times 10^{6}$ with a maximum injection time of $100 \mathrm{~ms}$. The top three most intense peaks in the MS1 with $z>2$ were selected for HCD fragmentation with a normalized collision energy setting of 25. The MS2 resolution was 120,000, the isolation window was $4 \mathrm{~m} / z$ units, and three microscans were averaged. Dynamic exclusion was enabled with a duration of $30 \mathrm{~s}$. Source-induced dissociation was set to $15.0 \mathrm{eV}$. One biological replicate and two technical replicates were performed for this analysis, generating 22 raw data files.

\section{Intact-Mass Data Deconvolution}

Intact-mass raw files were deconvoluted into monoisotopic components using Protein Deconvolution 4.0 software (Thermo Fisher Scientific) (minimum $\mathrm{S} / \mathrm{N}=2$, minimum number of detected charge states $=2$, fit factor $=70 \%$, remainder threshold $=10 \%$, target average spectrum width $=0.18 \mathrm{~min}$, target average spectrum offset $=34 \%$ ). Different charge state ranges were selected for deconvoluting different fractions: +5 to +30 for fractions $1-9$ and +5 to +50 for fractions $10-11$. Each raw file was split into three to nine retention time (RT) ranges for deconvolution so that the output tables did not exceed allowed spreadsheet size. In the subsequent mass calibration process, calibrated deconvolution files for fractions 1 and 2 were not obtained due to insufficient data points. Therefore, fractions 1 and 2 were not analyzed further. 


\section{Proteoform Family Visualization with Description of Alterations}

The proteoform family figures shown in the main manuscript (Figures 4 and 6) were modified slightly from the default Cytoscape output to eliminate false connections and improve clarity. In the Figure 4 bottom-right family (L28 family built with multi-protease G-PTM-D database), an edge (32 Da) between the 15,760.9 $\mathrm{Da}$ and 15,792.8 Da proteoforms was removed, as the new annotation of the 15,792.8 $\mathrm{Da}$ proteoform with the multi-protease G-PTM-D database is no longer a doubly-oxidized version of the 15,760.9 Da proteoform. In Figure 6 family A, a 10,720.8 Da proteoform was removed, as it was connected to the 10,752.8 Da proteoform with a 32 Da mass difference, but the latter did not contain two oxidations. In families A, C, and D, the edges between gene names and top-down proteoforms were removed for clarity. In both Figures 4 and 6, the slope of the linear function of node size vs. proteoform intensity has been adjusted for example families to enhance contrast.

*Previous publications by the Smith Group also contain helpful descriptions of experimental methods for proteoform identification. ${ }^{5-9}$

\section{RAW MS DATA FILES AND G-PTM-D DATABASES}

All raw data files and G-PTM-D databases are available on the MassIVE platform (MSV000083768, $\mathrm{ftp}: / /$ massive.ucsd.edu/MSV000083768, and MSV000083304, ftp://massive.ucsd.edu/MSV000083304). There are $66 \mathrm{MS}$ files (in .raw format) from NeuCode-labeled intact-mass proteomics (11 Gelfree fractions of 3 biological replicates with 2 technical replicate injections each). There are $22 \mathrm{MS}$ files from label-free top-down proteomics (11 fractions of one biological replicate with 2 technical replicate injections each). There are also $65 \mathrm{MS}$ files from multi-protease bottom-up proteomics (10 fractions for trypsin and 11 fractions for each of the other five proteases). The pruned trypsin-only G-PTM-D database and the pruned multi-protease G-PTM-D database built from these bottom-up data are also included. The intact-mass data, top-down data, and G-PTM-D databases can be found in data set MSV000083768. The bottom-up data can be found in data set MSV000083304.

\section{SUPPLEMENTARY RESULTS}

\section{NeuCode Intact-Mass-Only Analysis Using Unpruned Multi-Protease G-PTM-D Database}

We performed a Proteoform Suite analysis with calibrated NeuCode intact-mass data but using the full multi-protease G-PTM-D database instead of the pruned database. The full G-PTM-D database contained a large number of protein sequences $(\sim 61,000)$ and PTMs that were not detected in our bottom-up data, which expanded the search space significantly. The full G-PTM-D database was used to construct a theoretical proteoform catalog that contained 426,170 entries, which is 3.5 times larger than pruned database-derived catalog. Using the same data filtering parameters and selecting the same ET and EE peaks, 561 unique proteoforms were identified in this analysis at 30\% FDR (selecting peaks with the same low FDRs as those in the pruned database-assisted analysis was not possible). This high FDR is a result of the large search space of the theoretical proteoform catalog, which contributed to high FDRs in ET pairs (the highest being 134\%). This analysis illustrates how using a pruned G-PTM-D database can help to prevent high FDRs during proteoform identification.

\section{Integrated Intact-Mass/Top-Down Analysis Using Uncalibrated Data}

We performed a Proteoform Suite analysis using uncalibrated intact-mass and top-down data with a catalog generated from the pruned multi-protease G-PTM-D database. Raw mass components from these uncalibrated data were filtered with the same parameters reported in the main manuscript. ET 
comparisons revealed that only 103 pairs grouped to the 0.0185 Da peak ( $10 \%$ FDR), the only peak indicating exact matches. This is only $12 \%$ of the 863 exact-matching pairs in the analysis with calibrated data, leading to many fewer identifications in this analysis. Therefore, mass calibration is crucial to effectively identify proteoforms.

\section{Top-Down-Only Analysis Utilizing Top-Down MS1 Spectra as “Label-Free Intact-Mass" Data} We attempted to maximize the utility of the proteomics data collected, so we delved deeper into our labelfree top-down data to look for unidentified proteoforms observed in the MS1 spectra. This data analysis strategy was previously employed in yeast and murine mitochondrial proteoform analyses. ${ }^{8-9}$

Precursor ion spectra (MS1) of the top-down data files were extracted. These new top-down MS1-only files were referred to as "label-free intact-mass data files". They were deconvoluted like NeuCode intactmass data in Thermo Protein Deconvolution 4.0 to provide label-free raw mass components. The resultant mass components were calibrated as described in the main manuscript. Processed intact-mass data were imported into Proteoform Suite together with calibrated top-down hits. A theoretical proteoform catalog was constructed using the same multi-protease G-PTM-D database and the "patch" databases as described in the main manuscript, except only one PTM was allowed instead of four to prevent high FDRs in the subsequent ET comparison stage. NeuCode pair determination and lysine count calculation were skipped, so ET and EE comparisons proceeded with aggregated proteoform masses. ET and EE pairs were grouped with smaller intervals $(0.005$ and $0.01 \mathrm{Da}$, respectively), so that mass difference peaks with relatively low FDRs would be revealed and accepted for family construction.

The label-free intact-mass data further increased the number of unique proteoform identifications beyond those revealed by the integrated NeuCode intact-mass/label-free top-down analysis discussed in the main manuscript. In this label-free intact-mass/top-down analysis, Proteoform Suite identified 880 proteoforms representing 444 genes. The overall FDR for proteoform identification was $5.4 \%$, which is low considering the high noise level of ET and EE comparisons in label-free proteoform analysis (where we do not have the knowledge of lysine count to help limit the number of comparisons). We identified 169 unique intact-mass experimental proteoforms not found in the top-down hits. Among those, 120 proteoforms were new identifications not found in the NeuCode intact-mass proteoform identifications. These proteoforms represented 28 new genes not found in the previous analysis. These new identifications contributed to a $10 \%$ increase in total unique proteoform identifications, and a 5.8\% increase in total genes represented, yielding a final result of 1,327 $(1,207+120)$ proteoforms representing $512(484+28)$ genes. Over $45 \%$ of the proteoform identifications came from intact-mass measurements (NeuCode-labeled and/or label-free).

\section{Protein Entries in the Patch Database}

The patch database contained 101 protein accession numbers. Fifty-six of these accessions had peptidelevel evidence in the multi-protease bottom-up data, but the peptide of interest was observed above a $1 \%$ FDR, and therefore the accession was not included in the pruned multi-protease G-PTM-D database. Furthermore, 29 of the 101 accessions in the patch database were included as isoforms. The original UniProt XML database used to search the bottom-up data did not include isoform sequences, which explains why these 29 accessions were not included in the pruned multi-protease G-PTM-D database. That being said, peptide-level evidence for the canonical form of 26 of these proteins was observed in the bottom-up data (FDR $>2 \%$ ). We posit that many of the remaining 16 accessions in the patch database were eliminated from the pruned multi-protease G-PTM-D database as a result of the protein inference process. 


\section{Histone Proteoforms and PTMs}

Histone proteoforms are known to play important roles in gene expression. In this study, we identified 289 histone proteoforms among the 1,207 reported. These histone proteoforms contained PTMs including methylation, acetylation, phosphorylation, malonylation, succinylation, oxidation, deamidation, and carbamylation. Many of these PTM types play key roles in histone-mediated modulation of gene expression. There were 6 histone proteoform families assembled, including 5 ambiguously identified families and 1 unambiguously identified family (SI Table S-10). Ambiguous histone families account for half of the ambiguous families reported (5 out of 10), and this ambiguity is largely the result of the similar molecular weights of numerous types of histones $\mathrm{H} 2 \mathrm{~A}, \mathrm{H} 2 \mathrm{~B}$, and $\mathrm{H} 3$ (e.g., $\mathrm{H} 2 \mathrm{~B}$ type 1-A, B, C, etc.). It is important to note that, although most histone proteoforms were in the ambiguous families, many of them were still unambiguously identified through direct connections to known theoretical histone proteoforms. The one unambiguous histone family is the Histone $\mathrm{H} 3.1 \mathrm{t}$ proteoform family, containing 3 top-down and 3 intact-mass experimental proteoforms (SI Figure S-6)

\section{SUPPLEMENTARY FIGURES}
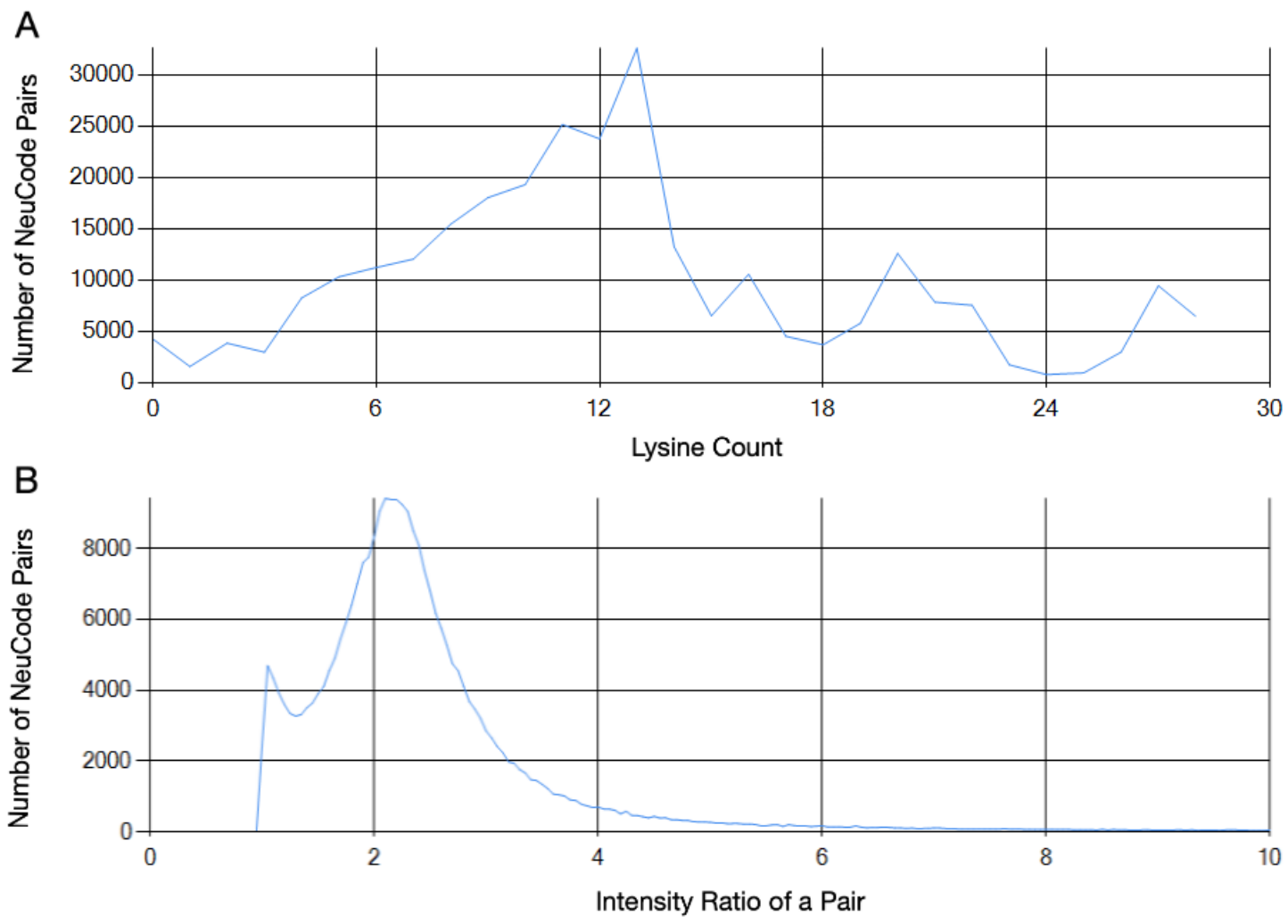

SI Figure S-1. Lysine count (A) and intensity ratio (B) distributions of the 283,634 NeuCode pairs that Proteoform Suite identified in this study. The peak intensity ratio was $2.15: 1$, which was close to the mixing ratio of "light" and "heavy" protein samples at 2:1. NeuCode pairs whose intensity ratios were between 1.8:1 to 2.5:1 were accepted for aggregation into experimental proteoforms. 


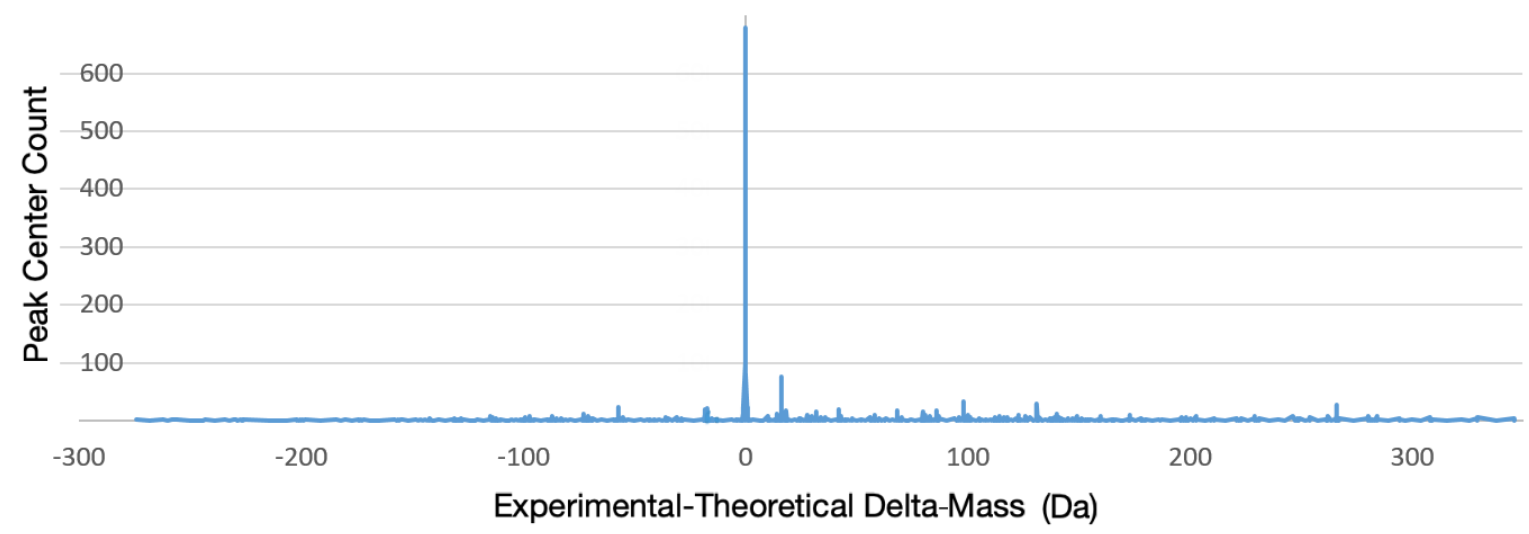

SI Figure S-2. Mass difference histogram from experimental-theoretical proteoform comparisons. Analysis was performed using intact-mass and top-down data with a catalog of theoretical proteoforms derived from the pruned multi-protease G-PTM-D database.

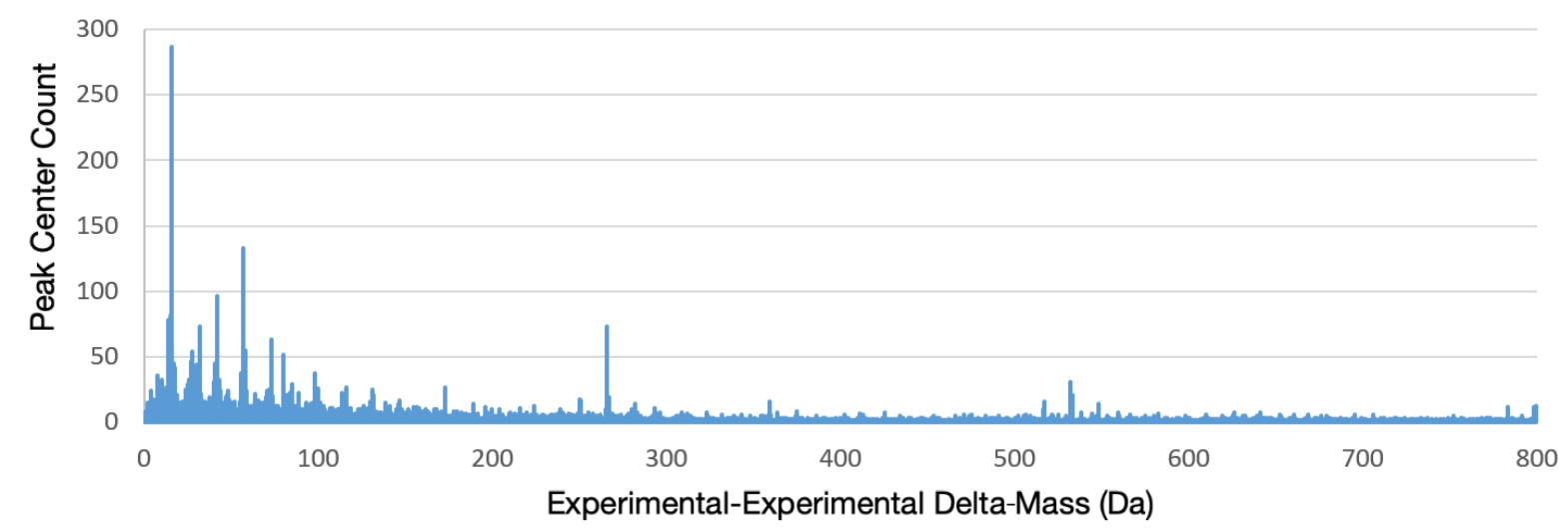

SI Figure S-3. Mass difference histogram from experimental-experimental proteoform comparisons. Analysis was performed using intact-mass and top-down data.

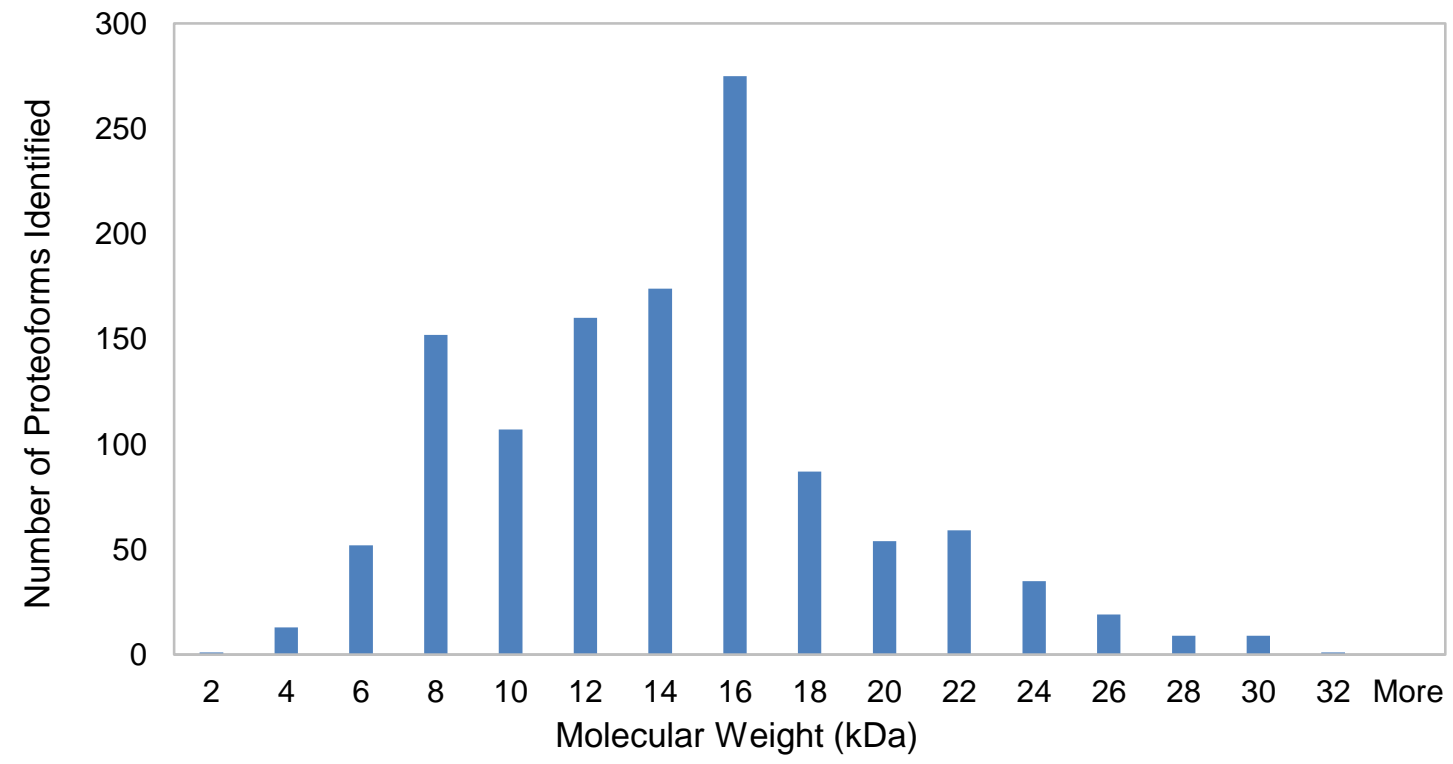

SI Figure S-4. Molecular weight distribution of the 1,207 identified proteoforms. This result is from the integrated intact-mass/top-down analysis. 


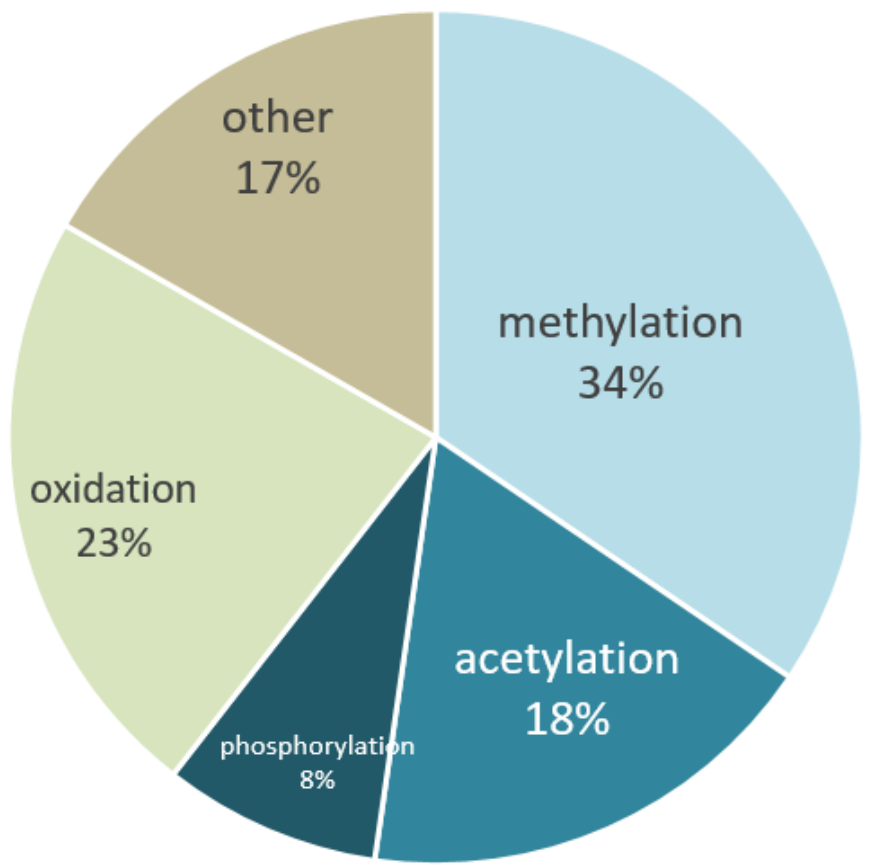

SI Figure S-5. PTM types found in the 1,207 identified proteoforms. This result is from the integrated intact-mass/top-down analysis.

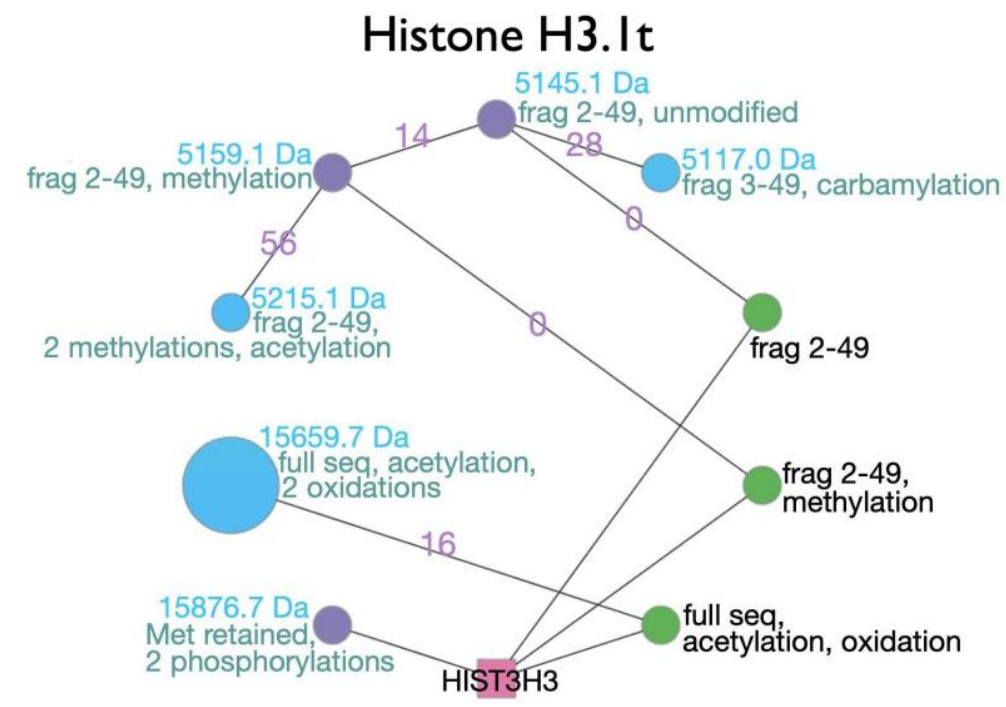

SI Figure S-6. Histone H3.1t proteoform family. Proteoforms in this family contain PTMs such as methylation, acetylation, and phosphorylation that are known to contribute to histone function. 


\section{REFERENCES FOR SUPPORTING INFORMATION}

(1) Wessel, D.; Flügge, U. I. A method for the quantitative recovery of protein in dilute solution in the presence of detergents and lipids. Anal. Biochem. 1984, 138, 141-143.

(2) Sheynkman, G. M.; Shortreed, M. R.; Frey, B. L.; Scalf, M.; Smith, L. M. Large-scale mass spectrometric detection of variant peptides resulting from nonsynonymous nucleotide differences. $J$. Proteome Res. 2014, 13, 228-240.

(3) Sheynkman, G. M.; Shortreed, M. R.; Frey, B. L.; Smith, L. M. Discovery and mass spectrometric analysis of novel splice-junction peptides using RNA-seq. Mol. Cell. Proteomics 2013, 12, 2341-2353.

(4) Miller, R. M.; Millikin, R. J.; Hoffman, C. V.; Solntsev, S. K.; Sheynkman, G. M.; Shortreed, M. R.; Smith, L. M. Improved Protein Inference from Multiple Protease Bottom-Up Mass Spectrometry Data. $J$. Proteome Res. 2019, 18, 3429-3438.

(5) Shortreed, M. R.; Frey, B. L.; Scalf, M.; Knoener, R. A.; Cesnik, A. J.; Smith, L. M. Elucidating Proteoform Families from Proteoform Intact-Mass and Lysine-Count Measurements. J. Proteome Res. 2016, 15, 1213-1221.

(6) Dai, Y.; Shortreed, M. R.; Scalf, M.; Frey, B. L.; Cesnik, A. J.; Solntsev, S.; Schaffer, L. V.; Smith, L. M. Elucidating Escherichia coli Proteoform Families Using Intact-Mass Proteomics and a Global PTM Discovery Database. J. Proteome Res. 2017, 16, 4156-4165.

(7) Cesnik, A. J.; Shortreed, M. R.; Schaffer, L. V.; Knoener, R. A.; Frey, B. L.; Scalf, M.; Solntsev, S. K.; Dai, Y.; Gasch, A. P.; Smith, L. M. Proteoform Suite: Software for Constructing, Quantifying, and Visualizing Proteoform Families. J. Proteome Res. 2018, 17, 568-578.

(8) Schaffer, L. V.; Shortreed, M. R.; Cesnik, A. J.; Frey, B. L.; Solntsev, S. K.; Scalf, M.; Smith, L. M. Expanding Proteoform Identifications in Top-Down Proteomic Analyses by Constructing Proteoform Families. Anal. Chem. 2018, 90, 1325-1333.

(9) Schaffer, L. V.; Rensvold, J. W.; Shortreed, M. R.; Cesnik, A. J.; Jochem, A.; Scalf, M.; Frey, B. L.; Pagliarini, D. J.; Smith, L. M. Identification and Quantification of Murine Mitochondrial Proteoforms Using an Integrated Top-Down and Intact-Mass Strategy. J. Proteome Res. 2018, 17, 3526-3536. 